\title{
BioHyTec: Biohybride Technologien in der Hauptstadtregion - Kompetenzbildung und Aufbau einer regionalen Wertschöpfungskette
}

\author{
Christian Vogt, Fred Lisdat
}

\section{InnoRegio}

\subsection{Der InnoRegio-Wettbewerb}

Das Bundesministerium für Bildung und Forschung (BMBF) startete 1999 mit dem InnoRegio-Wettbewerb eine neuartige Förderinitiative unter der Leitidee „Innovative Impulse in den Neuen Ländern“. In zahlreichen Regionen wurden Aktivitäten in Gang gesetzt, um neue Formen der Zusammenarbeit von Menschen aus den unterschiedlichsten Bereichen zu entwickeln und damit die Wertschöpfung und Wettbewerbsfähigkeit in den ostdeutschen Regionen zu erhöhen. An dieser Ausschreibung nahmen in der Anfangsphase 444 Bewerberregionen teil. Nach der ersten Jury-Sitzung im Oktober 1999 wurden 50 InnoRegios ausgewählt, in einer Entwicklungsphase ihre Kernkompetenzen herauszufiltern und tragfähige Innovationskonzepte zu erarbeiten. Mit der zweiten Jury-Sitzung im Herbst 2000 fiel der Startschuss zur Umsetzungsphase. Zur Zeit werden vom BMBF 23 InnoRegios in den Neuen Ländern gefördert.

\subsection{Das InnoRegio-Konzept von BioHyTec}

Zu diesen erfolgreichen InnoRegios gehört das Netzwerk BioHyTec mit seinem Konzept „Biohybrid-Technologien in der Region Potsdam-Luckenwalde“. Gemeint sind Technologien, die biologische Systeme und technische Bauelemente zu neuen Funktionseinheiten, wie z. B. Biochips oder künstlichen Organen, zusammenfuihren. Schwerpunkte der BioHyTec-Initiative sind dabei analytische Systeme zum Stoffnachweis. Basierend auf dem in der Region bestehenden Potenzial an Forschungseinrichtungen und einer Vielzahl von Unternehmen sieht das Konzept den Aufbau einer Wertschöpfungskette mit einer Fokussierung auf die Marktsegmente medizinische Diagnostik und Lebensmittelanalytik vor. Auf der Basis der Markteinschätzungen von 1999 [1] wurde das Technologiefeld Biochips zu den Zukunftsmärkten mit dem höchsten Wachstumspotenzial gezählt. Das Segment der high-density DNA-Chips für die Genom- und Pharmaforschung wurde von US-Firmen wie Affymetrix bereits besetzt und nicht als ein Zielmarkt identifiziert.

In der Umsetzungsstrategie wurden fünf Stoßrichtungen formuliert, die auf die Entwicklung einer Wertschöpfungskette in der Region zielen:

- Forschung und Entwicklung in Verbundprojekten zwischen Wissenschaft und Wirtschaft (regionale Biotech- und Diagnostikfirmen in Kooperation mit Forschungseinrichtungen) mit einer klaren Produktorientierung;
- Entwicklung einer breiten Produktpalette zum Teil kompatibler Biochips auf einer begrenzten Geräteplattform durch die konsequente Nutzung und Umsetzung des bei den Firmen und Forschungseinrichtungen vorhandenen biotechnischen Know-hows;

- Aufbau und Betrieb eines gemeinsamen Biochip-Centers (BCC) als Inkubator und zentrale technologische Schnittstelle beim Fraunhofer-Institut für Biomedizinische Technik in Bergholz-Rehbrücke (IBMT) für eine beschleunigte Vorlaufforschung; das BCC soll personell und apparativ so ausgestattet sein, dass es im „Rapid prototyping“-Verfahren Entwicklungsaufträge der Partner realisieren kann;

- regionale Ausbildungsoffensive mit einer Ausbildung zum „Biologielaboranten“ sowie ergänzend dazu einem dualen Studiengang „Biosystemtechnik/ Bioinformatik";

- organisatorische Strukturen in Form eines „Vereins für Bioanalytik und Biohybrid-Technologien, BioHyTec e. V.“, der alle Akteure zusammenfuihrt, einer Netzwerkkoordination mit einem Steuerungsgremium und einer Geschäftsstelle sowie einem Kuratorium (Aufsichtsrat) fuir das Controlling der Projekte.

BioHyTec steht im Rahmen des InnoRegio-Programms bis 2006 ein individuelles Budget von 8,2 Mio. € zur Verfügung. Dies ist deutlich weniger als in der ursprünglichen Konzeption geplant und steckt den Rahmen, innerhalb dessen selbstverantwortlich Projekte in den Bereichen Forschung und Entwicklung, Bildung sowie Öffentlichkeitsarbeit und Netzwerkmanagement entwickelt werden können.

\section{Status der InnoRegio-Initiative BioHyTec}

\subsection{Phase I}

Nach dem offiziellen Start der Umsetzungsphase wurden zuigig die organisatorischen Strukturen geschaffen bzw. angepasst, um das InnoRegio-Projekt in die Arbeitsphase zu überfuihren. So erhielt das erste Verbundprojekt bereits ein halbes Jahr später den Zuwendungsbescheid und nahm seine Arbeit auf. Vorausgegangen war eine intensive Evaluation durch das Kuratorium, das insbesondere die Innovation sowie die wirtschaftlichen Perspektiven der Projekte einzuschätzen hat und die Einbindung der beantragten Projekte in das Konzept der Region prüft. In dieser Phase I ab 2001 wurden insgesamt sechs Projekte gestartet, die im Zuge der InnoRegio-Konzeptentwicklung bereits einen fortgeschrittenen Planungsstand erreicht hatten. 


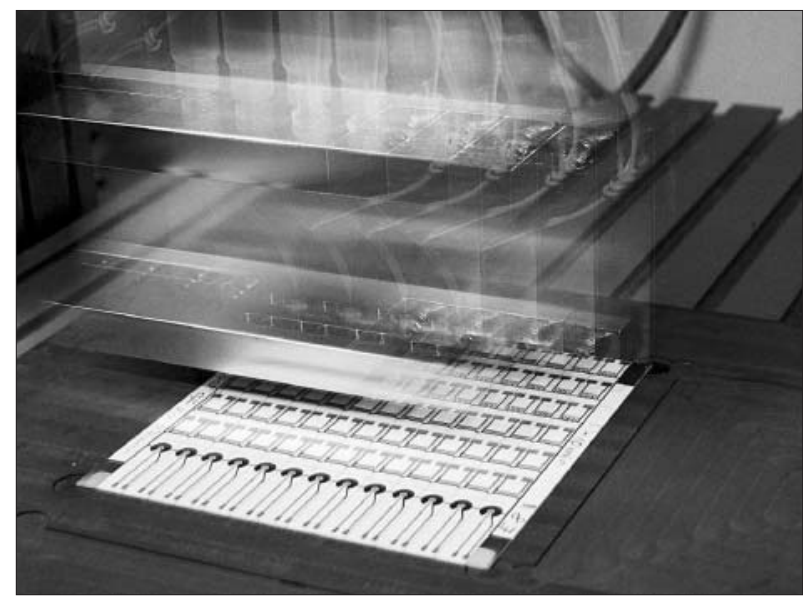

FILT-Präparation von Dickschichtelektroden für Messungen im Atemkondensat (FILT GmbH)

Foto: Harald Hirsch

Das Projekt „Mykotoxinchip“ mit 4 Verbundpartnern unter Projektleitung der BioTeZ Berlin-Buch GmbH zielt auf die Entwicklung eines Schnelltestes auf der Basis eines Biochips für die Detektion verschiedener Schimmelpilzgifte in Nahrungsmitteln. Das Marktpotenzial ist aufgrund des verschärften Haftungsrechtes der Lebensmittelhersteller weltweit gewachsen.

„Nichtinvasive Diagnostik“, ein Verbundprojekt unter Leitung der FILT GmbH mit fünf Partnern, verfolgt das Ziel, auf der Basis eines patentierten Atemkondensatsammlers spezifische Lungenerkrankungen zeitnah, ohne einen Eingriff in den Patienten und nach Möglichkeit vor Ort beim Facharzt nachzuweisen. Das System wird mit der derzeit verfuigbaren Analytik bisher v. a. in Europa verkauft. Mit einer Ausweitung der diagnostischen Möglichkeiten steigen die weiteren Chancen für die Erschließung neuer Märkte.

Die Entwicklung eines „Sensorchips für den Nachweis der antioxidativen Kapazität" wird unter Projektleitung der IGV GmbH in einem Verbundprojekt mit fünf Partnern verfolgt. Kosmetikhersteller sind EU-weit dazu verpflichtet, das beworbene antioxidative Schutzpotenzial ihrer Produkte nachzuweisen. Dieser Nachweis muss im Endprodukt, also der Creme oder Lotion, und nicht nur in den einzelnen Komponenten geführt werden. Die Verhandlungen mit führenden Kosmetikherstellern wurden bereits in der Phase der Projektentwicklung begonnen.

Ein Schnellnachweissystem für die Früherkennung von Geflügelkrankheiten auf der Basis eines DNS-Biochips wird im Projekt „Poultrychip“ unter Projektleitung der CONGEN Biotechnologie GmbH mit dem Fraunhofer Institut für Biomedizinische Technik (FhG-IBMT) entwickelt. Wichtige Viruserkrankungen von Geflügel sollen in einem - möglichst vor Ort einsetzbaren - preiswerten und schnellen Testsystem diagnostiziert werden können, um die Tiergesundheit zu erhalten und Massenschlachtungen vorbeugen zu können.

In Ergänzung zu diesen FuE-Verbundprojekten wurde bereits zum Wintersemester 2001 der „Duale Studiengang Biosystemtechnik/Bioinformatik“ an der Technischen Fachhochschule Wildau aufgebaut, der zunächst in einem dreijährigen Studium zum international anerkann- ten Abschluss als Bachelor führt. Zielstellungen dieses Bildungsprojektes sind die enge Verzahnung von Ausbildung und Wirtschaft, die Bereitstellung von qualifiziertem Fachpersonal für die Unternehmen der Region und die Schaffung von Zukunftsperspektiven in der Region für junge Menschen. Durch mehrwöchige Praktika pro Semester in den Unternehmen und Forschungseinrichtungen des Verbundes, durch die Zusammenarbeit mit den Unternehmen bei der Gestaltung der Studienpläne und durch die Vergabe von Abschlussarbeiten, die auf die Problemstellungen in den Unternehmen abgestellt sind, wurde der Studiengang bisher sowohl von den Studenten als auch von den Unternehmen als erfolgversprechend bewertet. Im diesem Sommer können die ersten Bachelor-Absolventen in der Region ihre Berufstätigkeit aufnehmen. Durch die Ausrichtung des Studiums auf Bioinformatik und Biosystemtechnik kann ein flexibler Einsatz auf einem sehr bewegten Markt gewährleistet werden.

Die InnoRegio-Geschäftsstelle übernahm beim Knotenpunkt des Netzwerkes, dem Verein BioHyTec e. V., das Projektmanagement und -controlling, die Außendarstellung auch auf Messen und organisierte den wissenschaftlichen Informationsaustausch in zahlreichen Workshops und auf Symposien.

\subsection{Phase II}

In der Phase II wurden ab Sommer 2002 neue Projekte generiert, die weitere Ansätze für die Integration von Nachweisverfahren auf einer Sensoroberfläche bieten

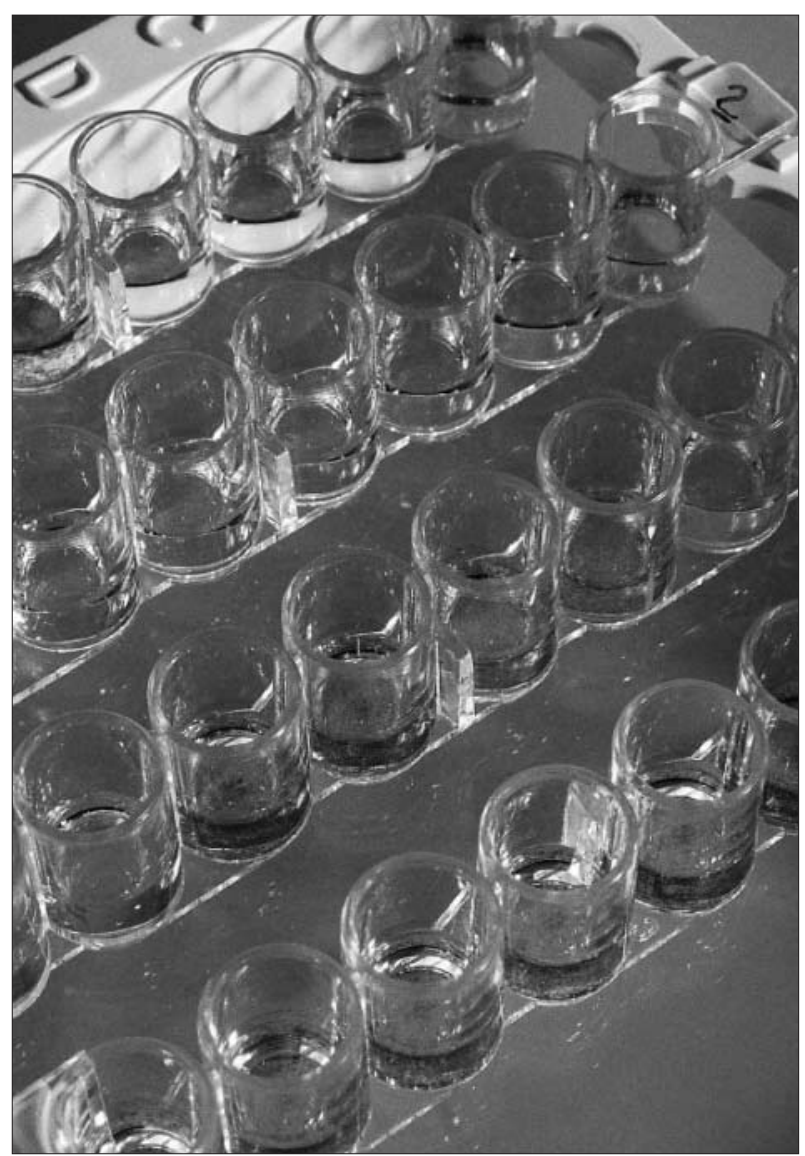

Ein Vorteil des Einsatzes von Biosensoren und Biochips besteht in der Messung einer großen Anzahl von Proben und geringen Probenvolumina (CONGEN GmbH).
Foto: Harald Hirsch 


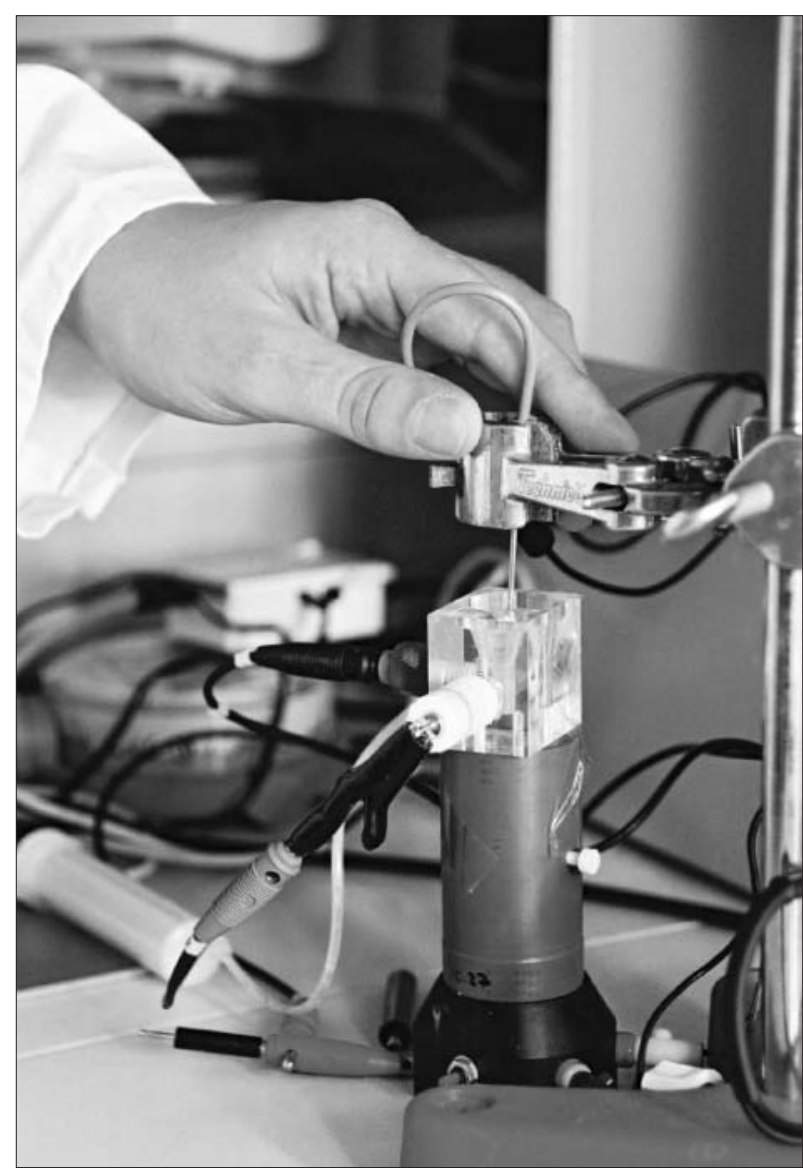

Sensormesszelle an der Universität Potsdam

Foto: Harald Hirsch

und das Know-how in der Region verbreitern. Die Projektlaufzeit liegt wiederum bei ca. drei Jahren, so dass diese Projekte im Jahr 2006 enden werden.

Das Verbundprojekt „Amplifikations- und Detektionssystem" unter der Projektleitung der AGOWA GmbH mit drei Verbundpartnern verfolgt die Zielstellung einer technologischen Weiterentwicklung von Biochips in Richtung „Lab-on-the-chip“. Erreicht werden soll dies durch die Integration von Probenvorbereitung, Amplifikation und Detektion. Neben dem technologischen Fortschritt soll damit ein deutlicher Wettbewerbsvorteil erzielt werden.

Unter Leitung der CellTrend GmbH aus Luckenwalde wird mit drei Verbundpartnern ein „Telomerasechip“ entwickelt. In diesem Verbundprojekt wird ein medizinischdiagnostischer Biochip entwickelt, der die Untersuchung von Tumorbiopsien auf erhöhte Telomeraseaktivität ermöglicht. Diagnostisch ist dieser Parameter für die genaue Charakterisierung des Entwicklungsstadiums von Krebszellen wichtig. Die derzeit mit einem hohen zeitlichen, personellen und technischen Aufwand betriebenen Untersuchungen sollen so massiv vereinfacht und auch fuir Krankenkassen bezahlbar gemacht werden.

Das junge Unternehmen AptaRes AG, ebenfalls aus Luckenwalde, entwickelt mit vier Verbundpartnern einen neuartigen „Diabeteschip“. Das Projekt eröffnet neue Perspektiven für die Untersuchung und Verlaufskontrolle einer diabetischen Stoffwechselsituation. Anhand eines einzelnen Markers kann nicht nur wie bisher die aktuelle Blutzuckerkonzentration des Patienten festgestellt werden, sondern der Spiegel für die vergangenen zwei bis drei Wochen, was eine präzisere medikamentöse Einstellung des Patienten mit weniger Risiken und Nebenwirkungen erlaubt. Auf dem weltweit stark umkämpften Markt kann mit diesem Projekt für die beteiligten Unternehmen ein Wettbewerbsvorteil erarbeitet werden.

Die Firma peptides \& elephants $\mathrm{GmbH}$, eine Ausgründung aus dem Deutschen Institut für Ernährungsforschung, entwickelt gemeinsam mit dem FhG-IBMT einen „Peptidchip“. Ziel des Projektes ist die Entwicklung eines Diagnostik-Chips für den Nachweis von Virusantikörpern im Serum. Damit wird die Produktpalette des Unternehmens um die Herstellung von in-vitro-Diagnostika erweitert.

Seit 2003 ist die InnoRegio-Geschäftsstelle mit dem gleichen Aufgabenbereich beim Fraunhofer-Institut für Biomedizinische Technik angesiedelt. Höhepunkt der organisatorischen Arbeiten war die Ausrichtung des dritten Deutschen Biosensorsymposiums Ende März 2003 in Potsdam. Diese nationale Tagung mit internationalen Gästen widmet sich aktuellen Entwicklungstendenzen der Bioanalytik mit dem Fokus Biosensorik/Biochips und findet alle zwei Jahre statt.

Für den weiteren Verlauf der InnoRegio-Initiative ist geplant, die BioHyTec-Aktivitäten mit anderen regionalen Initiativen auf dem Gebiet der Biotechnologie enger zu verknuipfen. So soll die Geschäftsstelle ab Juli 2004 mit BioTOP, dem Aktionszentrum für Biotechnologie der beiden Bundesländer Berlin und Brandenburg zusammengefuihrt werden, um die Vernetzungseffekte in der Region zu optimieren.

\subsection{Ergänzende Aktivitäten}

Mit den genannten Verbundprojekten konnten der regionalen Entwicklung wichtige Impulse gegeben werden. Es sind jedoch aufgrund des Umfangs der Projekte weitere Aktivitäten notwendig, um die angestoßene Kompetenzbildung auf bioanalytischem Gebiet in der Region zu verstetigen.

So wurden im Netzwerk weitere Projekte für die Region eingeworben, die das InnoRegio-Konzept unterstiitzen. Das ursprünglich als Teil der Ausbilungsoffensive entwickelte Projekt einer Ausbildung zum Biologischen Assis-

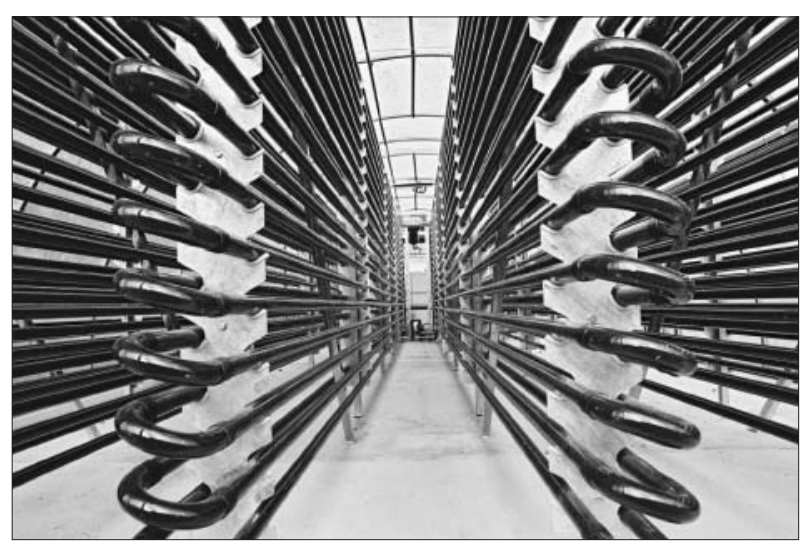

Produktionsanlage zur Gewinnung von Wirksubstanzen aus Algen; die Charakterisierung der Wirksamkeit erfolgt mit Biosensorchips (IGV GmbH). Foto: Harald Hirsch 


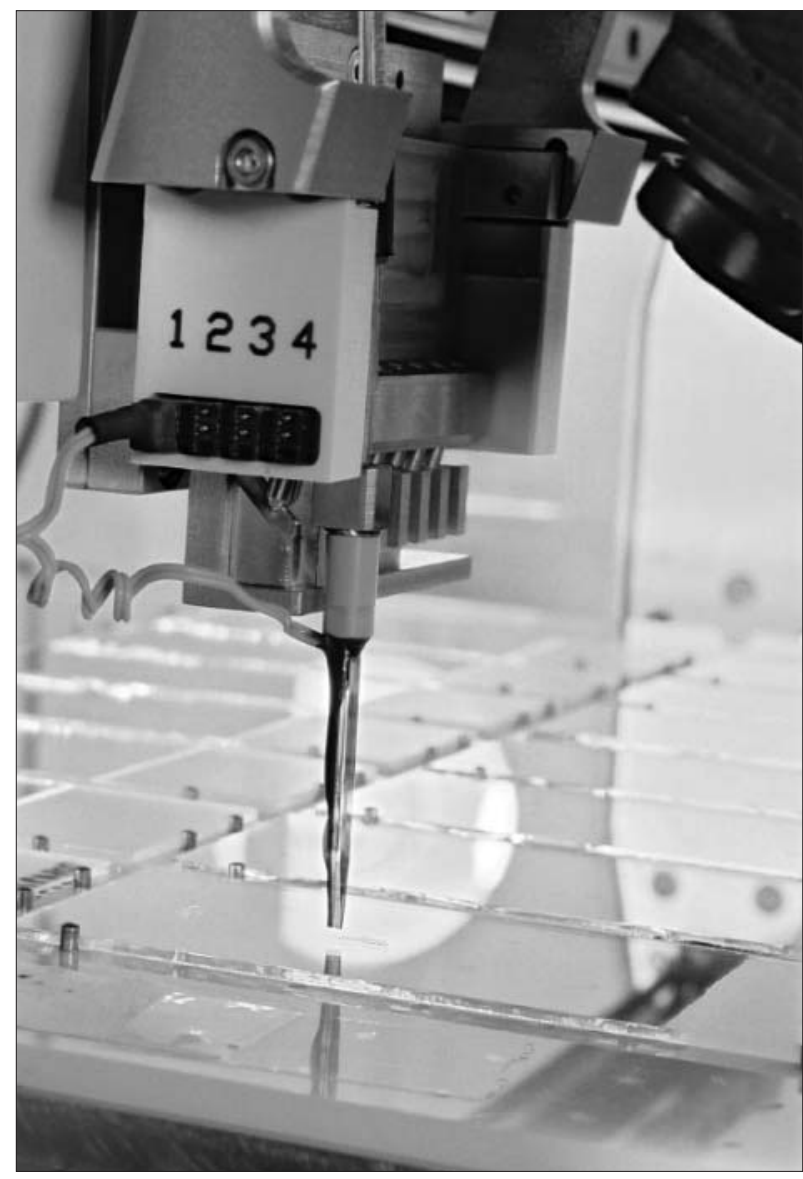

Spotter zur Beschichtung von Biochips auf der Basis von Glasträgern (FhG-IBMT)

Foto: Harald Hirsch

tenten wurde im Biotechnologiepark Luckenwalde mit EU-Fördermitteln realisiert, um Facharbeiter für die Unternehmen in der Region zu qualifizieren. Damit konnte eine Lücke in der Ausbildungskette geschlossen werden, die mit InnoRegio-Mitteln nicht realisierbar war.

Entsprechend der langfristigen Ausbildungsplanung laufen seit Mitte 2003 verstärkte Anstrengungen der Technischen Fachhochschule Wildau, den Bachelor-Studiengang durch einen Master-Studiengang zu komplettieren. Dieser aufbauende Masterstudiengang soll ab September 2004 den besten Bachelor-Absolventen die Möglichkeit bieten, einen dem Universitätsdiplom vergleichbaren, ebenfalls international anerkannten akademischen Abschluss zu erhalten und damit auch verhindern, dass die Absolventen die Region dauerhaft verlassen.

Das Ausbildungsprogramm, das sich die InnoRegio-Initiative vorgenommen hatte, ist damit umgesetzt. Für alle Ausbildungsebenen wurden regionale Angebote geschaffen, die angenommen und genutzt werden. Durch die enge Vernetzung von Ausbildung und Praxis wurde ein Angebot geschaffen, das auch in der Wirtschaft eine hohe Akzeptanz findet und die Berufseinstiegschancen der Absolventen wesentlich erhöht. Besonderer Wert wurde auf die heute zunehmend geforderten Schnittstellenkompetenzen gelegt. Biosystemtechnik arbeitet an den Überlappungsflächen von Biologie, Physik und Chemie, erfordert mathematisches Wissen und technologisches Verständnis. Bioinformatisches Wissen wird heute in vielen biotechnologischen Unternehmen und Forschungseinrichtungen vorausgesetzt und in Zukunft zu einer Schlüsselkompetenz werden.
Als zentraler technologischer Kristallisationspunkt und Kompetenzzentrum wurde am Fraunhofer-Institut für Biomedizinische Technik in Bergholz-Rehbrücke das BiochipZentrum BCC etabliert. Erste Investitionen für die Vorbereitung der Kleinserienproduktion von Biochips wurden durch das BMBF-Programm Innovations- und Gründerlabore maßgeblich unterstuitzt. Das BCC ist damit apparativ und personell so ausgestattet, dass es Entwicklungsaufträge - vom Biochip-Prototypen bis zur kompletten Anwenderlösung - erfuillen kann. Die Keimzelle für eine Serienproduktion ist damit geschaffen.

Neben dieser anwendungsbezogenen Forschung wurde im Netzwerk auch Vorlaufforschung betrieben. Schwerpunkte dieser Arbeit, v. a. an der Universität Potsdam, waren Markierungsstrategien von Antikörpern, Proteinelektroden sowie die Nutzung von DNS für die Bioanalytik. Die Forschungen wurden zum Teil durch das Ministerium für Wissenschaft, Forschung und Kultur des Landes Brandenburg gefördert.

Durch die Kooperation mit anderen Netzwerken der Region sind Synergieffekte entstanden. Weitere FuEProjekte im Biochip-Format werden z. B. im Nutrigenomik-Netzwerk aus der BMBF-Ausschreibung BioProfile gefördert. Dort ist ein Allergen-Chip in Arbeit. Für einen Adipositas-Biochip (Nachweis der genetischen Veranlagung fuir Fettleibigkeit) und einen SNP-Chip (Nachweis von Punktmutationen in bestimmten Genabschnitten) haben die Projektarbeiten begonnen. Ein InfektionsChip wird am FhG-IBMT mit dem Robert-Koch-Institut in Berlin entwickelt, ein Tuberkolose-Biochip, ein Hormon-Biochip sowie ein Drogen-Biochip befinden sich in der Entwicklung.

Generelle Zielstellung ist die Erhöhung der Anwendungsbreite von chipbasierten Nachweissystemen. Damit sollen sowohl die technologischen Basisplattformen gestärkt als auch das technologische Know-how in der Region insgesamt erweitert werden. Nicht zuletzt bedeutet dies für die Firmen eine Verbreiterung der Produktpalette.

\section{Aussichten}

Mit diesen Aktivitäten hat die Region ein breites Portfolio an Biochipentwicklungen angelegt. Im Rahmen der staatlichen Unterstuitzung können jedoch nur vorwettbewerbliche Entwicklungen gefördert werden. Das bedeutet, dass nach dem Abschluss der Projekte noch keine fertigen Produkte verfuigbar sind und sein dürfen - von einer Serien- oder gar Massenproduktion ganz abgesehen. In der Region hat sich aber eine Kompetenz aufgebaut, die sich in einer Produktpipeline für vielfältige Anwendungen zeigt, deren wirtschaftliche Auswirkungen sukzessive ab 2005/6 spürbar werden dürften.

Bis zu einer erfolgreichen Vermarktung der Produkte ist es noch ein weites Stiuck Weg. Eine Reihe von Faktoren werden einen großen Einfluss darauf haben, ob, wie, wo und wann die Produkte vermarktet werden können. Die entscheidende Einflussgröße sind die Markterfordernisse. Im Forschungsbereich, v. a. in der Genom- und 
Proteomforschung, hat sich die Biochip-Technologie etabliert. Dieses Marktsegment, das derzeit ca. 90 \% des Gesamtmarktes ausmacht, wird von wenigen Unternehmen, insbesondere den Biochip-Pionieren aus den Vereinigten Staaten, dominiert. Ausgefeilte und breit angelegte Patentstrategien des Marktfuihrers Affymetrix machen diesen Markt fuir neue Mitbewerber unattraktiv.

Anwendungen für Biochips außerhalb dieses Bereiches sind ebenfalls erfolgversprechend. Im Netzwerk BioHyTec werden in erster Linie Anwendungen für Analytik und Diagnostik entwickelt. Dieser Markt hat sich entgegen den Prognosen von Analysten und Marktforschern noch nicht mit der erwarteten Dynamik entwickelt. Eine ganze Palette von Faktoren erschwert hier die Entfaltung des Marktes. Analytik und Diagnostik, insbesondere die medizinische Diagnostik, sind ein stark regulierter Markt, der von gesetzlichen Vorgaben abhängig ist. Was muss, was kann getestet werden, welche Grenzwerte werden festgelegt? Das sind Fragen, die in der Öffentlichkeit erörtert, auf politischer Ebene diskutiert, in Gesetze gegossen und in Durchfuihrungsbestimmungen detailliert klargelegt werden müssen. Welche Höchstmengen an Acrylamid sind fuir den Menschen noch akzeptabel? Auf welche Schimmelpilzgifte müssen Nahrungsmittel getestet werden? Welche Aussagen zur Förderung der Gesundheit sind auf Lebensmitteln oder Kosmetika zugelassen und wie müssen sie getestet werden? Aber vor allem auch, inwieweit Krankenkassen die Kosten für diagnostische Tests übernehmen bzw. ob Patienten die Kosten für die eigene Prävention zu zahlen bereit sind, sind Rahmenbedingungen, die sich nur langsam verschieben lassen. Gesetzliche Regelungen werden der Etablierung dieser Technologie entscheidend Vorschub leisten.

Die Biochip-Technologie hat ihre Vorteile gegenüber den herkömmlichen Testverfahren in der Miniaturisierung und Parallelisierung. Nur bei einer hohen Menge an parallelen Tests bzw. verschiedener zu testender Parameter sind diese Vorzüge auszuspielen. Dann wird die Biochip-Technologie den herkömmlichen Methoden und Verfahren der Analytik gleichwertig sein und an ihre Seite treten können. Ein Traum, an dessen Umsetzung intensiv gearbeitet wird, sind Rundum-Biochips, das heißt Multi-Chips, die möglichst viele Allergene erfassen oder möglichst viele Krebsarten in einem frühen Stadium diagnostizieren. Damit wären die Vorteile dieser Technologie auch für den Anwender sehr augenscheinlich. Daneben wird es viele einfache Tests auf „festen Trägern“ geben, deren Anwendungsbreite sich heute kaum abschätzen lässt.

Ein wichtiger Schritt auf dem Weg der Technologieetablierung wird eine Standardisierung der Biochips und der Auswertegeräte sein. Für einen Test die Chips und das Auswertesystem zu kaufen, ist nicht für alle Anwender attraktiv. Technologische Plattformen, die eine breite Palette von Anwendungen für einen spezifischen Markt anbieten können, werden langfristig erfolgreich sein.

Neben der Marktentwicklung und der Ausreifung der Technologie sind auch Standortfaktoren entscheidend, ob sich das Netzwerk BioHyTec langfristig und nachhal- tig als Kompetenz-Cluster national und international etablieren kann. Das wissenschaftliche Potenzial in der Region ist vorhanden. Die Hauptstadtregion gehört zu den fuihrenden Biotech-Standorten in Deutschland und liegt hinsichtlich der Anzahl der Unternehmen und Forschungseinrichtungen sogar an erster Stelle. Diese wissenschaftliche Exzellenz ist ein wichtiger Standortfaktor, der auch für internationale Investoren eine Sogwirkung hat. Dafür müssen die Anstrengungen einer offensiven Vermarktung intensiviert werden. Besonders interessant und wichtig fur Investoren ist aber die enge Vernetzung von Wissenschaft und Wirtschaft, $\mathrm{z}$. T. auch über die Fachgrenzen hinweg. Hier leisten die Technologiefeld-Netzwerke wie BioHyTec, Nutrigenomik, Tissue Engineering, aber vor allem BioTOP als Aktionszentrum für Biotechnologie der Länder Berlin und Brandenburg einen Beitrag. Dieser Pluspunkt der Region muss weiter gestärkt und vermarktet werden. Mit dem neuen BiotopAktionsprogramm und einem Marketingkonzept fuir die Biotechnologie sind Instrumente erarbeitet worden, die nun in enger Abstimmung mit den Akteuren der Region und der Politik umgesetzt werden müssen.

Zu den Schwerpunktfeldern der Region in der Biotechnologie gehören auch die biohybriden Technologien, die hier eine längere Tradition haben. Der europaweit erste Biosensor fuir die Blutzuckerdiagnostik wurde hier zur Marktreife entwickelt und die ersten Geräte kamen bereits 1982 auf den Markt. Dieser Spitzenplatz konnte bis heute gehalten werden. Im Rahmen einer weltweiten Evaluation des World Technology Evaluation Center im Auftrag US-amerikanischer Regierungsbehörden [2] wurde das Netzwerk BioHyTec mit dem InnoRegio-Konzept unter den 20 weltweit führenden Arbeitsgruppen in Bioanalytik, Biosensorik und Molekularbiologie gelistet.

Mit den Forschungs- und Entwicklungsprojekten des InnoRegio-Verbundes und einer ganzen Bandbreite weiterer Projekte sind biohybride Technologien bzw. Systeme entwickelt worden, die nun aus der Phase der vorwettbewerblichen Entwicklung in die Serienproduktion überführt werden müssen. Zugleich besteht die Herausforderung, weitere Produkte zu entwickeln, um in der Region eine breite Produktpipeline aufzubauen und mittelfristig bis 2006 eine kritische Masse anzureichern, die eine Eigendynamik entwickelt. Wie wird das Netzwerk BioHyTec diese Aufgaben lösen?

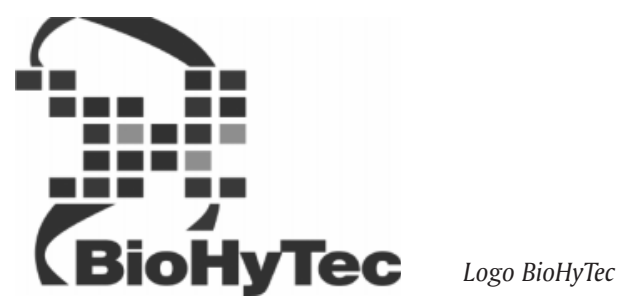

In erster Linie wird der Schwerpunkt in der Etablierung neuer Verbünde gesehen, die unter der Leitung von regionalen Firmen an produktorientierten Systemen arbeiten. So plant BioHyTec im Rahmen der letzten Phase der InnoRegio-Förderung ein weiteres Entwicklungsprojekt „Immunchip“ unter Leitung eines brandenburgischen 
Unternehmens, das über das zugesagte Förderbudget hinaus ein Biochip-System entwickeln wird, das eine schnelle Analytik von Autoimmunerkrankungen des menschlichen Körpers zulässt.

Als eine neue Etappe wird eine Bewerbung im BMBFFörderprogramm „Innovative regionale Wachstumskerne" vorbereitet. Aufsetzend auf den Ergebnissen und Erfahrungen aus InnoRegio steht eine Ausrichtung der Entwicklungsarbeiten auf wirtschaftliche Erfordernisse diesmal noch stärker im Vordergrund. Ziele sind die Erweiterung der Produktpalette in der Region, die Entwicklung einer kritischen Masse an biohybriden Systemen sowie die Vorbereitung einer wirtschaftlichen Umsetzung.

Die Vorstellungen über ein Produktionsunternehmen für Biochips haben sich im Projektverlauf mehrfach geändert, aber die Konturen werden langsam schärfer. Innerhalb des Netzwerkes hat sich ein Konsortium gebildet, das auf der Basis eines Makrochips arbeitet und nach Ablauf der FuE-Projekte im Sommer 2004 eine Überführung in die Serienproduktion plant. Hier werden neue Projekte akquiriert, die auf dieser Makrochip-Plattform arbeiten werden, um ab 2006 eine Serienproduktion - voraussichtlich in einem neuen Unternehmen, das im Land Brandenburg angesiedelt sein wird - aufzunehmen.

Parallel wird eine Mikrochip-Technologie entwickelt, die als Plattform den Projektpartnern zur Verfuigung steht und für die ebenfalls ergänzende Projekte akquiriert werden. Eine Kleinserienproduktion ist derzeit am BCC bereits möglich. Bei einer tragfähigen Auslastung ist eine Ausgründung angestrebt.

Durch die Einbindung von größeren Unternehmen wie der BRAHMS AG aus Hennigsdorf und der Scienion AG aus Berlin-Adlershof, einer Ausgründung aus dem MPI für Molekulare Genetik, werden Partner in das Netzwerk einsteigen, die über eigene Technologien und Vertriebskanäle verfuigen. Ob und inwieweit diese größeren Anbieter Produktionsaufgaben der forschenden KMUs bzw. auch die Vermarktung übernehmen, bleibt abzuwarten. Die Unternehmen des Netzwerkes, die einen Forschungsfokus haben, werden sich in den kommenden Jahren entscheiden müssen, ob und wie weit sie selbst den Aufbau einer eigenen Produktion und eines eigenen Vertriebs vorantreiben können oder wollen und welche Kooperationen sie eingehen.

Wie es in drei bis vier Jahren auch aussehen mag, die Chancen stehen gut, dass biohybride Technologien in der Region zu einem Wirtschaftsfaktor geworden sind.

\section{Anmerkungen/Literatur}

[1] NEXUS-Report 1998, Biolnsights 1999, Frost \& Sullivan 1999

[2] World Technology Evaluation Center im Auftrag von National Institutes of Health, National Science Foundation, National Aeronautics and Space Administration, US Department of Agriculture, US Army Research Office 2004

\section{Autoren}

Christian Vogt M.A.

BioHyTec e. V.

Verein für Bioanalytik und Biohybridtechnologien

c/o Universität Potsdam

Karl-Liebknecht-Str. 24-25

14476 Golm

Tel. +493320088587

E-Mail:vogt@biohytec.de

\section{Prof. Dr. Fred Lisdat}

Technische Fachhochschule Wildau

Fachbereich Ingenieurwesen/Wirtschaftsingenieurwesen BioHyTec e. V.

Tel. +49 3375 508-456, +49 331 977-5127

E-Mail: flisdat@igw.tfh-wildau.de 